International Journal of Geometric Methods in Modern Physics (C) World Scientific Publishing Company

\title{
T-duality Invariance of the Supermembrane
}

\author{
María Pilar García del Moral \\ Departamento de Física, Universidad de Oviedo, Avda Calvo Sotelo S/n. Oviedo,33007 España\} \\ garciamormaria@uniovi.es \\ Joselen Peña \\ Departamento de Física, Facultad de Ciencias, \\ Universidad Central de Venezuela, A.P. 47270, Caracas 1041-A, Venezuela \\ jpena@ciens.fisica.ucv.ve \\ Alvaro Restuccia \\ Departamento de Física, Universidad de Antofagasta, Aptdo 02800, Chile \\ \& Departamento de Física, Universidad Simón Bolívar \\ Apartado 89000, Caracas 1080-A, Venezuela \\ arestu@usb.ve \\ Received (Day Month Year) \\ Revised (Day Month Year)
}

\begin{abstract}
We show that the supermembrane theory compactified on a torus is invariant under Tduality. There are two different topological sectors of the compactified supermembrane (M2) classified according to a vanishing or nonvanishing second cohomology class. We find the explicit T-duality transformation that acts locally on the supermembrane theory and we show that it is an exact symmetry of the theory. We give a global interpretation of the T-duality in terms of bundles. It has a natural description in terms of the cohomology of the base manifold and the homology of the target torus. We show that in the limit when the torus degenerate into a circle and the M2 mass operator restricts to the stringlike configurations, the usual closed string T-duality transformation between the type IIA and type IIB mass operators is recovered. Moreover if we just restrict M2 mass operator to string-like configurations but we perform a generalized T-duality we find the $\mathrm{SL}(2, \mathrm{Z})$ non-perturbative multiplet of IIA.
\end{abstract}

Keywords: Supermembrane, T-duality, M-theory, IIA, IIB String Theories.

\section{Introduction}

In this note we show that the supermembrane compactified on a torus is invariant under a generalized T-duality transformation [1. As a new result, we show how in the String Theory limit, the T-duality transformation for the supermembrane becomes the standard T-duality transformation of the closed superstrings compactified on a circle. The compactified supermembrane on a target space $M_{9} \times T^{2}$ may be formulated in terms of sections on symplectic torus bundles [2]. There are two well-defined sectors: one in which a topological condition due to an irreducible 
wrapping is imposed, that corresponds to the so-called supermembrane with central charges [5], and a second one without that restriction. While the first one can be globally formulated in terms of sections on symplectic torus bundles with $S L(2, \mathbb{Z})$ monodromy, the second corresponds to the formulation on trivial symplectic torus bundles [1. Physically the two sectors have very different properties, among which the most relevant one is that the regularized supermembrane with central charges has discrete spectrum [7] -so it is a well-defined quantum object- in distinction with the compactified supermembrane without that condition [6].

In 8 it was argued that a fundamental formulation of string/M-theory should exist in which the T- and U-duality symmetries are manifest from the start. In particular, it was argued that many massive, gauged supergravities cannot be naturally embedded in string theory without such a framework [9, 10, 11]. In [1] we showed that the supermembrane on a torus with central charges is in fact the origin of type IIB, and IIA gauged supergravities in $9 \mathrm{D}$. We showed the existence of a new $Z_{2}$ symmetry that plays the role of T-duality in the supermembrane interchanging the winding and $\mathrm{KK}$ charges but leaving the Hamiltonian invariant. T-duality becomes an exact symmetry of the symplectic torus bundle description of the supermembrane by fixing its energy tension.

\section{The Compactified Supermembrane in $M_{9} \times T^{2}$}

We consider now the compactified supermembrane embedded on a target space $M_{9} \times T^{2}$ where $T^{2}$ is a flat torus. We consider maps $X^{m}, X^{r}$ from $M_{9} \times T^{2}$ to the target space, where $X^{m}$ with $m=3, \ldots, 9$ are single valued maps onto the Minkowski sector of the target space while $X^{r}$, with $r=1,2$ maps onto the $T^{2}$ compact sector of the target. The winding condition corresponds to

$$
\oint_{\mathcal{C}_{s}} d X^{1}=2 \pi R\left(l_{s}+m_{s} R e \tau\right) ; \quad \oint_{\mathcal{C}_{s}} d X^{2}=2 \pi R m_{s} \operatorname{Im} \tau ; \quad \oint_{\mathcal{C}_{s}} d X^{m}=0
$$

where $R, \tau$ are respectively the radius and the Teichmuller parameters of the 2torus target-space, and $l_{s}, m_{s}, s=1,2$, are integers. The physical hamiltonian in the LCG is given by

$$
\begin{aligned}
& \mathcal{H}=\int_{\Sigma} T_{11}^{-2 / 3} \sqrt{W}\left[\frac{1}{2}\left(\frac{P_{m}}{\sqrt{W}}\right)^{2}+\frac{1}{2}\left(\frac{P_{r}}{\sqrt{W}}\right)^{2}+\frac{T_{11}^{2}}{2}\left\{X^{r}, X^{m}\right\}^{2}+\frac{T_{11}^{2}}{4}\left\{X^{r}, X^{s}\right\}^{2}\right] \\
& +\int_{\Sigma} T_{11}^{-2 / 3} \sqrt{W}\left[\frac{T_{11}^{2}}{4}\left\{X^{m}, X^{n}\right\}^{2}-\bar{\Psi} \Gamma_{-} \Gamma_{m}\left\{X^{m}, \Psi\right\}-\bar{\Psi} \Gamma_{-} \Gamma_{r}\left\{X^{r}, \Psi\right\}\right]
\end{aligned}
$$

subject to the constraints

$$
\begin{aligned}
& \phi_{1}:=d\left(\frac{P_{m}}{\sqrt{W}} d X^{m}+\frac{P_{r}}{\sqrt{W}} d X^{r}-\bar{\Psi} \Gamma_{-} d \Psi\right)=0, \\
& \phi_{2}:=\oint_{C_{s}}\left(\frac{P_{M}}{\sqrt{W}} d X^{M}+\frac{P_{r}}{\sqrt{W}} d X^{r}-\bar{\Psi} \Gamma_{-} d \Psi\right)=0,
\end{aligned}
$$

associated with a residual symmetry of the theory: the infinite group of diffeomorphims preserving the Riemann basis $\Sigma$. So far, we have described the compactified 
supermembrane with no distinction between sectors. We now impose an extra topological restriction on the winding maps [12]: the irreducible winding constraint,

$$
\int_{\Sigma} d X^{r} \wedge d X^{s}=n \epsilon^{r s} \operatorname{Area}\left(T^{2}\right) \quad n \neq 0, r, s=1,2
$$

where $\operatorname{Area}\left(T^{2}\right)=(2 \pi R)^{2} \operatorname{Im} \tau$.

-When this condition holds, $(n \neq 0)$ we refer to it as the supermembrane with central charge theory [5] and it implies that the winding matrix $\mathbb{W}=\left(\begin{array}{cc}l_{1} & l_{2} \\ m_{1} & m_{2}\end{array}\right)$ has $\operatorname{det} \mathbb{W}=n \neq 0$. Globally it corresponds to a sector, with nontrivial symplectic torus bundle with monodromy $\rho$ in $S L(2, Z)$, characterized by having $H^{2}\left(\Sigma, \mathbb{Z}_{\rho}\right) \neq 0$ [2], [1].

-When this condition vanishes $(n=0)$, this corresponds to a sector that we will call from now on compactified $\mathrm{M} 2, n=0$. Globally it corresponds to a trivial symplectic torus bundle over the base, lets choose for symplicity the flat 2-torus $\Sigma_{1}$. It is characterized by having a trivial class of $H^{2}\left(\Sigma_{1}, \mathbb{Z}\right)$.

The Mass operator of the compactified supermembrane with winding and KK contribution [4], [3], is

$$
\text { Mass }^{2}=T_{11}^{2}\left((2 \pi R)^{2} n I m \tau\right)^{2}+\frac{1}{R^{2}}\left(\left(m_{1}^{2}+\left(\frac{m|q \tau-p|}{R I m \tau}\right)+T_{11}^{2 / 3} H\right.\right.
$$

where the $H$ is defined in terms of the above hamiltonian $\mathcal{H}$ once the winding contribution has been extracted $H=\mathcal{H}-T_{11}^{-2 / 3} \int_{\Sigma} \sqrt{W} \frac{T_{11}^{2}}{4}\left\{X_{h}^{r}, X_{h}^{s}\right\}^{2}$ [4]. In the case of the sector of the compactified M2, sector $n=0$, the winding contribution vanishes.

\section{T-duality in the Supermembrane Theory}

In this section we introduce the T-duality transformations for the supermembrane theory [1]. This goes beyond the T-duality of superstring theory. The T-duality transformation we consider, is a nonlinear map which interchange the winding modes $\mathbb{W}$, associated to the cohomology of the base manifold with the KK charges, $Q=(p, q)$ associated to the homology of the target torus together with a transformation of the real moduli $R \rightarrow \frac{1}{R}$ and complex moduli $\tau \rightarrow \widetilde{\tau}$, both in a nontrivial way. In the following all transformed quantities under T-duality are denoted by a tilde.

In order to define the T-duality transformation we introduce the following [4](47) dimensionless variables

$$
\mathcal{Z}:=T_{11} A \widetilde{Y} \quad \widetilde{\mathcal{Z}}:=T_{11} \widetilde{A} Y
$$

where $T_{11}$ is the supermembrane tension, $A=(2 \pi R)^{2} \operatorname{Im} \tau$ is the area of the target torus and $Y=\frac{R I m \tau}{|q \tau-p|}$. The tilde variables $\widetilde{A}, \widetilde{Y}$ are the transformed quantities 
under the T-duality. See (7) for the explicit value of $\mathcal{Z}$. The T-duality transformation we introduce is given by [1]:

$$
\begin{aligned}
& \text { The moduli : } \quad \mathcal{Z} \widetilde{\mathcal{Z}}=1, \quad \widetilde{\tau}=\frac{\alpha \tau+\beta}{\gamma \tau+\alpha} \\
& \text { The charges }:\left(\begin{array}{l}
\widetilde{p} \\
\widetilde{q}
\end{array}\right)=\Lambda_{0}\left(\begin{array}{l}
p \\
q
\end{array}\right),\left(\begin{array}{cc}
\widetilde{l}_{1} & \widetilde{l}_{2} \\
\widetilde{m}_{1} & \widetilde{m}_{2}
\end{array}\right)=\Lambda_{0}^{-1}\left(\begin{array}{cc}
l_{1}^{\prime} & l_{2}^{\prime} \\
m_{1}^{\prime} & m_{2}^{\prime}
\end{array}\right) .
\end{aligned}
$$

With $\Lambda_{0}=\left(\begin{array}{ll}\alpha & \beta \\ \gamma & \alpha\end{array}\right) \in S L(2, Z)$. In the above definition the T-dual supermembrane corresponds to a new supermembrane where the role of winding and KK charges interchanged, i.e. the KK modes are mapped onto the winding modes $\left(\begin{array}{c}\widetilde{p} \\ \widetilde{q}\end{array}\right)=\left(\begin{array}{c}l_{1}^{\prime} \\ m_{1}^{\prime}\end{array}\right)$ and viceversa. The above property together with the condition $Z \widetilde{Z}=1$ ensure that $(\mathrm{T} \text {-duality })^{2}=\mathbb{I}$, the main property of T-duality. The explicit transformations of the real modulus, obtained from the above T-duality transformation is

$$
\widetilde{R}=\frac{|\gamma \tau+\alpha \| q \tau-p|^{2 / 3}}{T_{11}^{2 / 3}(\operatorname{Im} \tau)^{4 / 3}(2 \pi)^{4 / 3} R}, \text { with } \quad \widetilde{\tau}=\frac{\alpha \tau+\beta}{\gamma \tau+\alpha} \quad \text { and } \quad \mathcal{Z}^{3}=\frac{T_{11} R^{3}(\operatorname{Im} \tau)^{2}}{|q \tau-p|}
$$

The winding modes and KK charge contribution in the mass squared formula transform in the following way:

$$
T_{11} n^{2} A^{2}=\frac{n^{2}}{\widetilde{Y}^{2}} \mathcal{Z}^{2}, \quad \frac{m^{2}}{Y^{2}}=T_{11}^{2} m^{2} \widetilde{A}^{2} \mathcal{Z}^{2}
$$

To see how the $H_{1}$ (4) transforms under T-duality it is important to realize the transformation rules for the fields,

$$
d X^{m}=u d \widetilde{X}^{m}, \quad d \widetilde{X}=u e^{i \varphi} d X, \quad A=u e^{i \varphi} \widetilde{A} \quad \Psi=u^{3 / 2} \widetilde{\Psi}, \quad \bar{\Psi}=u^{3 / 2} \widetilde{\bar{\Psi}}
$$

Where $u=\mathcal{Z}^{2}=\frac{R|\gamma \tau+\alpha|}{\widetilde{R}}, \varphi$ a phase defined in (3.22) of [1] and $d X=d X^{1}+i d X^{2}$ and respectively, its dual $d \widetilde{X}$ is

$$
d \widetilde{X}=2 \pi \widetilde{R}\left[\left(\widetilde{m}_{1} \widetilde{\tau}+\widetilde{l_{1}}\right) d \widehat{X}^{1}+\left(\widetilde{m}_{2} \widetilde{\tau}+\widetilde{l}_{2}\right) d \widehat{X}^{2}\right]
$$

The phase $e^{i \varphi}$ cancels with the h.c. the transformation of the Hamiltonian. The relation between the hamiltonians through a T-dual transformation is

$$
H=\frac{1}{\widetilde{\mathcal{Z}}^{8}} \widetilde{H}, \quad \widetilde{H}=\frac{1}{\mathcal{Z}^{8}} H .
$$

We thus obtain for the mass squared formula the following identity,

$$
M^{2}=T_{11}^{2} n^{2} A^{2}+\frac{m^{2}}{Y^{2}}+T_{11}^{2 / 3} H=\frac{1}{\widetilde{\mathcal{Z}}^{2}}\left(\frac{n^{2}}{\widetilde{Y}^{2}}+T_{11}^{2} m^{2} \widetilde{A}^{2}\right)+\frac{T_{11}^{2 / 3}}{\widetilde{\mathcal{Z}}^{8}} \widetilde{H} .
$$




\subsection{T-Duality on Symplectic Bundles}

T-duality does not only acts at local level but also globally. As shown in [1] one can define an equivalence class with the elements of the coinvariant group associated to the monodromy group $G,\{\mathcal{Q}+g \widehat{\mathcal{Q}}-\widehat{\mathcal{Q}}\}$, such that for any $g \in G$ and $\widehat{\mathcal{Q}} \in H_{1}\left(T^{2}\right)$, it characterizes one symplectic torus bundle. In the formulation of the supermembrane on that geometrical structure, $\mathcal{Q}$ are identified with the KK charges. The action of $G$, the monodromy group, leaves the equivalence class invariant. We now consider the duality transformation introduced previously. Under the duality transformation the equivalence class transform as

$$
\{\mathcal{Q}+g \widehat{\mathcal{Q}}-\widehat{\mathcal{Q}}\} \rightarrow\left\{\Lambda_{0} \mathcal{Q}+\left(\Lambda_{0} g \Lambda_{0}^{-1}\right) \Lambda_{0} \widehat{\mathcal{Q}}-\Lambda_{0} \widehat{\mathcal{Q}}\right\}
$$

hence for the dual bundle it holds, $\left\{\Lambda_{0}\left(\begin{array}{c}l_{1} \\ m_{1}\end{array}\right)+\left(\Lambda_{0} g \Lambda_{0}^{-1}\right)\left(\begin{array}{c}\widehat{l_{1}} \\ \widehat{m_{1}}\end{array}\right)-\left(\begin{array}{c}\widehat{l_{1}} \\ \widehat{m_{1}}\end{array}\right)\right\}$, That is, as an element of the coinvariant group of $\Lambda_{0} G \Lambda_{0}^{-1}$. We then conclude that the duality transformation, in addition to the transformation on the moduli $R, \tau$, also maps the geometrical structure onto an equivalent symplectic torus bundle with monodromy $\Lambda_{0} G \Lambda_{0}^{-1}$. We notice that the transformation depends crucially on the original equivalence class of the coinvariant group. So for a nonequivalent symplectic torus bundle the dual transformations is realized with a different $S L(2, Z)$ matrix $\Lambda_{0}$. Now we are in position to determine the T-duality as a natural symmetry for the family of supermembranes with central charges. We take:

$$
\widetilde{Z}=Z=1 \Rightarrow T_{0}=\frac{|q \tau-p|}{R^{3}(\operatorname{Im} \tau)^{2}} .
$$

It imposes a relation between the energy scale of the tension of the supermembrane and the moduli of the torus fiber and that of its dual. Indeed we can think in two different ways: given the values of the moduli it fixes the allowed tension $T_{0}$ or on the other way around, for a fixed tension $T_{0}$, the radius, the Teichmuller parameter of the 2-torus, and the KK charges satisfy (14).

\section{String Theory Limit}

We then consider within the physical configurations of the supermembrane with central charges, the string-like configurations

$$
X^{m}=X^{m}\left(\tau, q_{1} \widehat{X}^{1}+q_{2} \widehat{X}^{2}\right), \quad A^{r}=A^{r}\left(\tau, q_{1} \widehat{X}^{1}+q_{2} \widehat{X}^{2}\right),
$$

where $q_{1}, q_{2}$ are relative prime integral numbers. $X^{m}, A^{r}$ are scalar fields on the torus $\Sigma$, a compact Riemann surface, hence they may always be expanded on a Fourier basis in term of a double periodic variable of that form. The restriction of $q_{1}, q_{2}$ to be relatively prime integral numbers arises from the global periodicity condition. On that configurations all the brackets

$$
\left\{X^{m}, X^{n}\right\}=\left\{X^{m}, A^{r}\right\}=\left\{A^{r}, A^{s}\right\}=0
$$


vanish. We then obtain the final expression for the mass contribution of the string states [4:

$$
\left.M_{11}^{2}\right|_{S C}=\left(n T_{11} A\right)^{2}+\left(\frac{m}{Y}\right)^{2}+8 \pi^{2} R_{11} T_{11}|q \tau-p|\left(N_{L}+N_{R}\right)
$$

where $(p, q)$ are relatively prime. We notice that $(p, q)$ may be interpreted as the wrapping of the membrane around the two cycles of the target torus. The corresponding change in the harmonic sector is 4

$$
d X_{h}=\left(q m d \widetilde{X}^{1}+p d \widetilde{X}^{2}\right)+\widetilde{\tau}\left(-Q n d \widetilde{X}^{1}+P d \widetilde{X}^{2}\right),
$$

the hamiltonian is invariant under that change. $p, q$ and $Q, P$ are now the winding numbers of the supermembrane. Given $p, q$ there always exist $Q$ and $P$ with the above property, although the correspondence is not unique. The $(p, q)$ type IIB strings may indeed be interpreted as different wrappings of the supermembrane with central charges. This nice interpretation was first given in [3].

The $(p, q) I I B$ string compactified on a circle of radius $R_{B}$ has tension [3]

$$
T_{(p, q)}^{2}=\frac{\left|q \lambda_{0}-p\right|^{2}}{\operatorname{Im} \lambda_{0}} T^{2}
$$

where $T=T_{11}^{2 / 3}$ is the string tension and $\lambda_{0}=\xi_{0}+i e^{-i \phi_{0}}$ with $\xi$ and $\phi$ identified with the scalar fields of the type IIB theory, $\phi$ corresponds to the dilaton fields. $\lambda_{0}$ is the asymptotic value of $\lambda$-the axion-dilaton of the type IIB theory- specifying the vacuum of the theory. The perturbative spectrum of the $(p, q)$ IIB string is [3],

$$
M_{B}^{2}=\left(\frac{n}{R_{B}}\right)^{2}+\left(2 \pi R_{B} m T_{(p, q)}\right)^{2}+4 \pi T_{(p, q)}\left(N_{L}+N_{R}\right) .
$$

If we use following [3] a factor $\beta^{2}$ to identify term by term both mass formulas $\left(M_{11}=\beta M_{B}\right)$, since there were obtained using different metrics, one gets

$$
\tau=\lambda_{0}, \quad \beta^{2}=\frac{T_{11} A_{11}^{1 / 2}}{T}, \quad R_{B}^{-2}=T T_{11} A_{11}^{3 / 2} .
$$

They were obtained by counting modes under some assumptions on the supermembrane wrapping modes, as mentioned on one of the footnotes [3. Here we have derived the expressions from a consistent definition of the supermembrane with central charges.

The identification of (17) to the mass formula of IIA string compactified on a circle of radius $R_{A}$ and tension $T_{A}$ may also be performed. In order to have a consistent identification one has to take $\operatorname{Re} \tau=0, p=1$ and hence $q=0$ in (17). The mass formula for the perturbative spectrum of type IIA is

$$
M_{A}^{2}=\left(\frac{m}{R_{A}}\right)^{2}+\left(2 \pi R_{A} n T_{A}\right)^{2}+4 \pi T_{A}\left(N_{L}+N_{R}\right)
$$

Identification after the limit process of the winding contributions and KK ones using a factor $(\beta \gamma)$ to compare the mass $^{2}$ formulas, since they are obtained using different metrics, yields $R_{A}=\beta \gamma R_{11}, \quad T_{A}=\gamma^{-2}(\operatorname{Im} \tau)^{1 / 2} T$ which imply

$$
\left(2 \pi R_{A} R_{B}\right)=\left(\frac{1}{T_{A} T_{(p, q)}}\right)^{1 / 2}
$$


We have thus obtained the $(p, q)$ IIB and IIA perturbative spectrum, when compactified on circles $R_{B}$ and $R_{A}$ respectively, from the string states on the supermembrane with central charges.

In this limit, by restricting the worldvolume configurations of the M2 to those of the string [4, we exactly recover the mass operator of the IIB theory as was formerly conjectured by Schwarz. The gain is that from the supermembrane with central charges the pure membrane excitations are known. If now a T-duality is performed on the supermembrane M2 mass operator restricted to string-like configurations, then an $\mathrm{SL}(2, \mathrm{Z})$ non-perturbative multiplet of IIA is obtained [4.

String T-duality transformation limit. Now we can take directly the limit of the T-duality transformations of the supermembrane to recover the standard T-duality transformations for the closed string operator. Before taking the limit it is convenient to consider a redefinition of $X^{1}$ and $X^{2}$. The coordinates that wrap on the $T^{2}$. We take $X^{1} \rightarrow \frac{X^{1}}{T_{11}^{1 / 6} R_{11}^{1 / 2}}, X^{2} \rightarrow T_{11}^{1 / 6} R_{11}^{1 / 2} X^{2}$, such that the Lie brackets $\left\{X^{1}, X^{2}\right\}^{2}$ will remain invariant under the redefinition. Now we consider the following limit for the torus collapsing into a circle, by imposing $R_{11} \rightarrow 0$. Since we want to ensure $A_{11} Y$ remains finite but $A_{11} \rightarrow 0$, necessarily $q=0$, for arbitrary $p$. Indeed this is equivalent to consider the KK charges $(p, q)=m(1,0)$ for $m=p$. By using the M2 T-duality transformation it can also be seen that $\tilde{A}_{11} \rightarrow 0$, so the dual also corresponds to a string. Moreover substituting in the previous definitions it can be seen $R_{A}$ is finite implies $R_{B}$ finite. We will define the following for the torus degenerating into a circle $S^{1}$

$$
R_{1}=\frac{R_{11}^{1 / 2}}{T_{11}^{1 / 6}} ; \quad R_{2}=R_{11}^{3 / 2} T_{11}^{1 / 6} \operatorname{Im} \tau
$$

Since $R_{11} \rightarrow 0$, it implies $R_{1} \rightarrow 0$ but $R_{2}$ is finite, so it corresponds to a closed curve that topologically is a circle. Now we re-express the winding condition in terms of the new variables. In terms of the new variables we get

$$
\oint_{\mathcal{C}_{s}} d X^{1}=2 \pi R_{1} l_{s} ; \quad \oint_{\mathcal{C}_{s}} d X^{2}=2 \pi R_{2} m_{s}
$$

Since $R_{1} \rightarrow 0$, although $l_{s}$ is taking finite, the first winding condition vanishes and the only residual winding condition is associated to the $S^{1}$ modulus is $R_{2}$. The former T-duality relations of the moduli in this limit become reduced to:

$$
Z \widetilde{Z}=\left.1\right|_{\text {string }}, \quad \Rightarrow \quad T_{M 2}^{4 / 3} R_{2}^{3} \widetilde{R}_{2}^{3}=1 \rightarrow \widetilde{R}_{2}=\frac{\alpha^{\prime}}{R_{2}} .
$$

where $\widetilde{R}_{2}=T_{11}^{1 / 2} \widetilde{A}^{1 / 2} \widetilde{Y}^{1 / 2}$. This defines for $T_{(p, q)}=T$ on the IIB string side precisely the duality relation of the strings. The transformation on the charges and windings are given by (6) and we finally obtain:

$$
\left\{R ;\left(l_{1}, m\right)\right\} \stackrel{T-\text { duality }}{\longrightarrow}\left\{\widetilde{R}=\frac{\alpha^{\prime}}{R} ;\left(m, l_{1}\right)\right\}
$$


Authors' Names

where $m$ is the common factor between the charges.

\section{Discussion and Conclusions}

We showed the existence of a new $Z_{2}$ symmetry that plays the role of T-duality in M-theory interchanging the winding and KK charges but leaving the hamiltonian invariant. The supermembrane compactified on a torus realizes this duality as an exact symmetry of the theory in both sectors $(n=0, n \neq 0)$. This is a relevant property expected for a sector of M-theory. When only string-like states are considered but it is performed this generalized T-duality of the supermembrane we obtain the mass operator IIB and the corresponding dual realizes the type IIA with all nonperturbative $S L(2, Z)$ multiplet. If we take the limit of the T-duality transformation of the supermembrane into the standard one, then only the standard $(p, q)=(1,0)$ mass type IIA operator is allowed meanwhile IIB mass operator is unchanged.

\section{Acknowledgements}

Part of the work of MPGM was funded by the Spanish Ministerio de Ciencia e Innovación (FPA2006-09199) and the Consolider-Ingenio 2010 Programme CPAN (CSD2007-00042). The work of A. R. is funded by Proyecto FONDECYT 1121103.

\section{References}

[1] M.P. Garcia del Moral, J.M. Pena, A. Restuccia, Supermembrane origin of type II gauged supergravities in 9D. JHEP 1209 (2012) 063.

[2] M.P. Garcia del Moral, I. Martin, J.M. Pena and A. Restuccia, SL(2,Z) symmetries, Supermembranes and Symplectic Torus Bundles, JHEP1109:068,2011.

[3] J.H. Schwarz, An SL(2,Z) multiplet of type IIB superstrings, Phys.Lett. B360 (1995) 13-18, Erratum-ibid. B364 (1995) 252.

[4] M.P. Garcia del Moral, I. Martin, A. Restuccia Nonperturbative SL(2,Z) (p,q)-strings manifestly realized on the quantum M2. arXiv:0802.0573.

[5] I. Martin, J. Ovalle and A. Restuccia, Compactified D = 11 supermembranes and symplectic noncommutative gauge theories. Phys.Rev. D64 (2001) 046001.

[6] B. de Wit, K. Peeters and J Plefka, Supermembranes with winding, Phys.Lett. B409 (1997) $117-123$.

[7] L. Boulton, M.P. Garcia del Moral, and A. Restuccia, Discreteness of the spectrum of the compactified $D=11$ supermembrane with nontrivial winding, Nucl.Phys.B671 (2003) 343-358.

[8] C. M. Hull, A geometry for non-geometric string backgrounds, JHEP 0510:065, 2005.

[9] J. Shelton, W. Taylor and B. Wecht, Nongeometric flux compactifications., JHEP 0510:085, 2005.

[10] C.M. Hull and R.A. Reid-Edwards, Flux compactifications of M-theory on twisted Tori, JHEP 0610 (2006) 086.

[11] H. Samtleben, Lectures on Gauged Supergravity and Flux Compactifications, Class. Quant. Grav. 25 (2008) 214002.

[12] I. Martin, A. Restuccia and Rafael S. Torrealba, On the stability of compactified $D=11$ supermembranes, Nucl.Phys. B521(1998) 117-128. 